\title{
Analysis of Performance Improvement in Wireless Sensor Networks Based on Heuristic Algorithms Along with Soft Computing Approach
}

\author{
Morteza Kabiri ${ }^{1}$, Javad Vahidi ${ }^{2}$ \\ ${ }^{1}$ Department of Computer, Islamic Azad University Ayatollah Amoli Branch, Amol, Iran, \\ Morteza.kabiri@gmail.com \\ ${ }^{2}$ Department of Applied Mathematics, Iran University of Science and Technology, Tehran, Iran, \\ jvahidi@iust.ac.ir
}

Article history:

Received July 2014

Accepted August 2014

Available online September 2014

\begin{abstract}
The use of Wireless Sensor Networks (WSNs) has grown dramatically in recent decades, and the use of these networks in the areas of military, health, environment, business, etc. increases every day. A wireless sensor network consists of many tiny sensor nodes with wireless communications and work independently. In applications of such sensor nodes, hundreds or even thousands of low-cost sensor nodes are dispersed over the monitoring area, in which each sensor node periodically reports its sensed data to the base station (sink). Due to limitations in the communication range, sensor nodes transmit their sensed data through multiple hops. Each sensor node acts as a routing element for other nodes for transmitting data.

One of the most important challenges in designing such networks is the management of energy consumption of nodes; because replacing or charging the batteries of these nodes are usually impossible.

One of the main characteristics of these networks is that the network lifetime is highly related to the route selection. Unbalanced energy consumption is an inherent problem in WSNs characterized by the multi-hop routing and many-to-one traffic pattern. This uneven energy dissipation in many routing algorithms can cause network partition because some nodes that are part of the efficient path are drained from their battery energy quicker. To efficiently route data through transmission path from node to node and to prolong the overall lifetime of the network, In this thesis we proposed three new routing algorithms using a combination of both Fuzzy approach and A-star algorithm seeks to investigate the problems of balancing energy consumption and maximization of network lifetime for WSNs :A-Star with 3 parameters fuzzy system $(\mathrm{A} * 3 \mathrm{~F})$, A-Star with 3 fuzzy system with 2 parameters using majority vote (A*3FMV) and A-Star with 3 fuzzy system with 2 parameters using simple additive weighting (A*3FSAW). The new methods is capable of selecting optimal routing path from the source node to the sink by favoring the highest remaining energy, minimum number of hops, lowest traffic load and energy consumption rate.

We evaluate and compare the efficiency of the proposed algorithms with each other methods under the same criteria in four different topographical areas. Simulation results show that A*3PFSAW and A*3PFMV balances the energy consumption well among all sensor nodes and achieves an obvious improvement on the network lifetime that randomly scattered nodes and flat routing..
\end{abstract}


Keywords: Wireless Sensor Networks, A-Star algorithm, Fuzzy logic, Network lifetime, Multi-hop routing.

\section{Introduction}

A wireless sensor network is a collection of nodes that form a network working together. Each node has a processing capability, memory, a transmitter / receiver RF, a unit of power (battery or solar cell) and can have different types of sensors are operating. After the nodes in a distributed environment, wirelessly communicate with each other and organize themselves into a contingency operation as a whole.

Since sensor networks can contain various types of sensors such as vibration sensor, magnetic, thermal, acoustic, visual and radar, so can monitor the various environmental conditions such as temperature, humidity, movement of vehicles, the lightning, the pressure, noise levels, the presence or absence of certain kinds of objects, mechanical pressure levels on the objects, properties of objects, such as current speed, direction and size. [1]

Sensor nodes can be continuously use for discovery event, a sense of place and local control. Features of micro-sensing and wireless communication between the nodes, promising many applications in the new fields of applications such as fields of military, health, home and business and categorized into the areas of space exploration, chemical treatments and relief for natural disaster.

Usually, sensor nodes are randomly distributed in the environment. The main components of communication are:

- Sensor nodes. Each of these nodes, the ability to collect and send data to the sink, wirelessly. Communicate with the sink nodes can be single-stage or multi-stage.

- The base station (sink) that communicates with the user via the Internet or satellite.

- Something that user wants to receive information about it.

- The user that data collected to measure / monitor the behavior of the phenomenon.

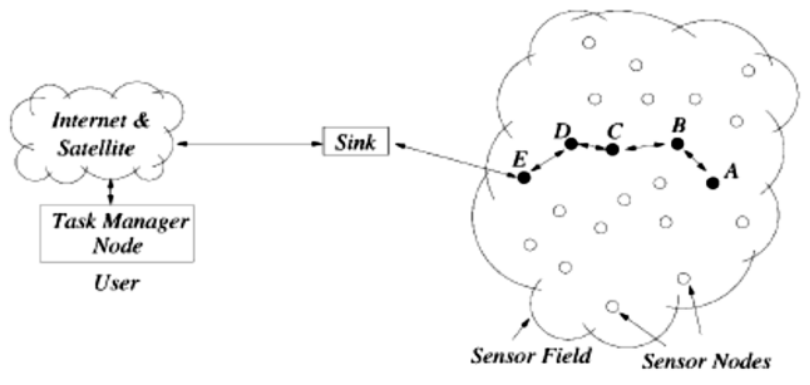

Fig.1. communication architecture for wireless sensor networks

In the past few years, intensive research on the potential of collaboration among sensors to collect and process sensed data and the coordination and management of activities are performed. However, sensor nodes have limited energy supply and bandwidth. Thus, innovative ways to eliminate inefficiencies in energy constraints that reduces the lifetime of the network is required.

Despite the innumerable applications of wireless sensor networks, these networks have several limitations, for example, limited energy supply, limited computing power, and limited bandwidth of wireless links. 


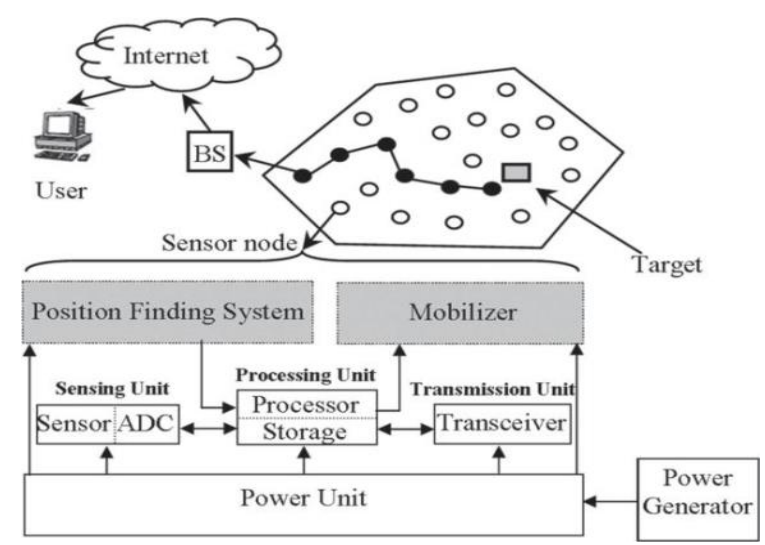

Fig.2. Components of a sensor node

One of the main goals of the design of wireless sensor networks Performing data communication while trying to prolong network lifetime and to prevent damage connection by applying energy management techniques. The design of routing protocols in wireless sensor networks is affected by many challenging factors. Before communication effectively in wireless sensor networks must overcome these factors. Some routing challenges and design issues that affect the routing of wireless sensor networks are deploying nodes, the energy consumption without loss of accuracy of the reported data, the heterogeneity of nodes and connections, fault tolerance, scalability, network dynamics, communication medium, density or density, coverage area, data integration, quality of service and ...[4].

Due to limitations in the communication range, sensor nodes transmit their sensed data through multiple hops. Each sensor node acts as a routing element for other nodes for transmitting data. Energy is therefore a crucial parameter in power-constrained data-gathering sensor networks. Energy consumption should be well managed to maximize the network lifetime [5]. Unbalanced energy consumption is an inherent problem in WSNs characterized by the multi-hop routing and many-to-one traffic pattern. The uneven energy dissipation can significantly reduce network lifetime. Generally in routing algorithm, the best path is chosen for transmission of data from source to the destination. Over a period of time, if the same path is chosen for all communications in order to achieve battery performance in terms of quick transmission time, then those nodes on this path will get drained fast [3], [5], [7].The problem with many algorithms is that they minimize the total energy consumption in the network at the expense of non-uniform energy drainage in the networks. Such approaches cause network partition because some nodes that are part of the efficient path are drained from their battery energy quicker.

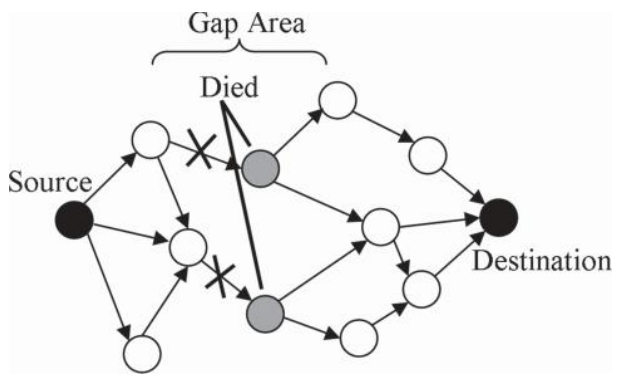

Fig. 3. Network partition due to the death of certain nodes 
The fuzzy inference system (FIS) can optimizes the routing path (depending on the metrics: distance, remaining battery power and energy consumption rate) in a distributed fashion. When a data is needed to be sent the protocol selects the optimal path through the FIS. Designers and developers of protocols and applications for WSN have emphasized on heuristic search technique, called A-Star algorithm, for searching best path for routing in WSN. They suggest that the criteria to search best path is not only to get path with minimum energy consumption but also to see that nodes selected in the path contain enough of residual energy.

Therefore, in this paper, the proposed method for balancing energy consumption and maximization of network lifetime for WSNs. We propose a new approach by combining Mixed-Fuzzy approach and A-star algorithm to select the optimal routing path from the source to the destination by favoring the highest remaining battery power, minimum number of hops ' minimum traffic loads and minimum energy consumption rate.

\section{RELATED WORKS}

Many challenges are in the design of wireless sensor networks such as energy efficiency, network scalability, network operating environment, the fault-tolerance, data delivery models, data integration, quality of service, delay, distribution of nodes, mobility or lack of mobility of nodes, the nodes are identical or not, network congestion, etc., which is one of the most prominent and important of these challenges, the problem of limited energy and how efficient it is to have a significant impact on how routing and a lot of research in this field is such that it can be cited, such as the following: for example the work in [8] proposed to minimize the hop stretch of a routing path (defined the shortest path) in order to reduce the energy cost of end-to-end transmission. The approaches in [9], [10] took a different view for prolonging the network-lifetime. They attempt to sustain the availability of the sensors that have less energy by distributing the traffic load to the ones with much residual energy. All of the above-mentioned works focus on improving energy-efficiency using fixed routing paths; nonetheless, due to the lack of path diversity, those nodes traversed by fixed routing paths may drain out their energy quickly.

The work in [11] exploited two natural advantages of opportunistic routing, i.e. path diversity and the improvement of transmission reliability, to develop a distributed routing scheme for prolonging the network lifetime of a WSN. The goal of this work is to assist each sensor in determining a suitable set of forwarders as well as their priorities, thus, enabling effort to extend the network-lifetime. Madan et al. in [12] solved the lifetime maximization problem with a distributed algorithm using the dual decomposition and the sub gradient method. Chang and Tassiulas in [13] proposed a shortest cost path routing algorithm for maximizing network lifetime based on link costs that reflect both the communication energy consumption rates and the residual energy levels. The authors of [14] presented a uniform balancing energy routing protocol to choose the nodes whose residual energies were greater than a certain threshold as routers for other nodes in every transmission round, and distributed the energy load among any sensors to maximize the whole network lifetime.

Lu et al. in [15] proposed an Energy-Efficient Multi-path Routing Protocol (EEMRP). It has the capability of searching multiple node-disjoint paths and utilizes a load balancing method to assign the traffic over each selected path. Both the residual energy level of nodes and the number of hops are considered to be incorporated into the link cost function. It uses a fairness index to evaluate the level of load balancing over different multi-paths. Furthermore, since EEMRP only takes care of data transfer delay, the reliability of successful paths sometimes is limited. The authors in [16] presented a new routing protocol based on a high weight genetic algorithm. In this method, the sensor nodes are aware of the data traffic rate to monitor the network congestion.

FML-MP (a fuzzy multi-path maximum lifespan routing scheme), an online multi-path routing scheme that strives to achieve a good distribution of the traffic load is developed in [17]. It uses an edge-weight function in the path search process. 
In [18] the authors presented Optimal Forwarding by Fuzzy Inference Systems (OFFIS) for flat sensor networks. The OFFIS protocol selected the best node from candidate nodes in the forwarding paths by favoring the minimum number of hops, shortest path and maximum remaining battery power, etc. The authors in [19] presented a novel algorithm for routing analysis in WSNs utilizing a fuzzy logic at each node to determine its capability to transfer data based on its relative energy levels, distance and traffic load to maximize the lifetime of the sensor networks.

Rana et al. in [20] used A-star algorithm to search optimal route from the source to destination in such a way that, there is a pre-defined minimum energy level for sensor nodes so that sensor node doesn't participate in routing if its residual energy level is below that level.

Deepak S. Gaikwad and Sampada Pimpale in [29] and have presented a combination protocol (A-Star with fuzzy) like such we have proposed, major weakness of this protocol considering only two input parameters residual energy level and the traffic load and they considered the time of death the first live nodes in the network without checking history of energy consumption rate at each node as base of improvement, which was summarized in comparison with the proposed protocols can be said that the time of death of the first node, the number of nodes remaining alive at the end of the scenarios and remaining energy in different algorithms are influenced by factors such as geographical location in the network, moving the BS, and the size of the network (length and width) and the network will behave differently, however, in a square network field, the proposed Gaikwad and Pimpale method, the time of death of the nodes be longer but in our proposed method by proper using of energy consumption rate (ECR) as third parameter for selecting optimal path the network lifetime is prolonged. Even using (ECR) in one of the proposed methods, the number of nodes alive at the end of the scenario which leads to higher levels of residual remaining energy.

In most applications of WSNs, sensor nodes are densely deployed in large areas. Once deployed, nodes can never be recharged or replaced. After depleting their energy, nodes turn to die and stop working. Since networks cannot accomplish assigned missions after nodes die [4], [6]. The maximization of lifetime can be formulated as an optimization problem. The variables of this optimization problem are routing parameters at nodes. When having sensed or asked to relay a data packet, each node needs to transmit this packet to a sink. However, it cannot send the packet directly to sinks except that it is a sink's neighbor. So normally a node needs to choose a neighboring sensor as its next hop. When nodes are chosen as the next hops they will influence the energy consumption of the network as well as the lifetime.

Energy Balanced Distributing in Routing is one of the solutions for maximize network lifetime and optimized management in energy consumption. WSN networks often suffer from the problem of using uneven energy, the unfavorable energy dissipation causes network lifetime of WSN can be severely reduced.

From the aforementioned literatures, we note that a number of different metrics have been used to prolong the lifetime of the sensor networks such as : Remaining Energy (RE) [3], [15], [21], Minimum Hop (MH) [15], [18], [19], [21] and Traffic Load (TL) [3], [16], [19], [21].

To extend the network lifetime, this paper proposes a new routing method using a combination of MixFuzzy approach and A-star algorithm. The proposed routing method is used to select the optimal routing path from source to destination by considering Remaining Energy, Minimum Hop, Traffic Load and Energy Consumption rate and balancing between them to lengthen the lifetime of the sensor network as much as possible.

\section{Fuzzy Approach}

Fuzzy logic was first introduced in the mid-1960s by Lotfi-Zadeh in [22]. Since then, its applications have rapidly expanded in adaptive control systems and system identification. It has the advantages of easy implementation, robustness, and ability to approximate to any nonlinear mapping. 
Fuzzy logic analyzes information using fuzzy sets, each of which is represented by a linguistic term such as "small," "medium," or "large." Fuzzy sets allow an object to be a partial member of a set. In Fig. 4, if X suggests a collection of objects denoted by $\mathrm{x}$, usually $\mathrm{X}$ is referred to as the "universe of discourse," and then a fuzzy set $\mathrm{A}$ in $\mathrm{X}$ is defined by a set of ordered pairs:

$\mathrm{A}=\{(\mathrm{x}, \mu \mathrm{A}(\mathrm{x})) / \mathrm{x} \in \mathrm{X}\}$.

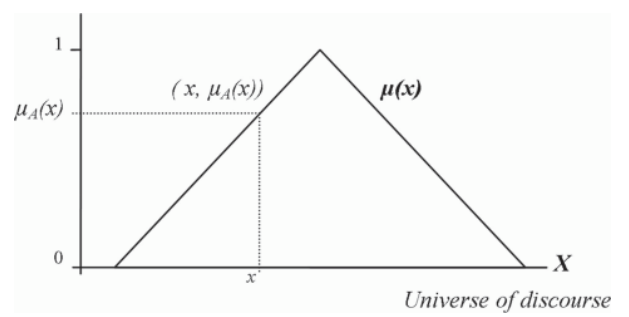

Fig. 4. Membership function from the pair $(x, \mu A(x))$.

Where the function $\mu \mathrm{A}(\mathrm{x})$ is called membership function of the object $\mathrm{x}$ in A. This membership function represents a "degree of belongingness" for each object to a fuzzy set, and provides a mapping of objects to a continuous membership value in the interval [0...1]. When a membership value is close to the value $1(\mu \mathrm{A}(\mathrm{x})-\rightarrow 1)$, it means that input $\mathrm{x}$ belongs to the set $\mathrm{A}$ with a high degree, while small membership values $(\mu \mathrm{A}(\mathrm{x})-\rightarrow 0)$, indicate that set $A$ does not suit input $\mathrm{x}$ very well [23].

In fuzzy systems, the dynamic behavior of a system is characterized by a set of linguistic fuzzy rules based on the knowledge of a human expert. Fuzzy rules are of the general form: If antecedent(s) then consequent(s), where antecedents and consequents are propositions containing linguistic variables. Antecedents of a fuzzy rule form a combination of fuzzy sets through the use of logic operations. Fig 5 shows the typical structure of a fuzzy system. It consists of four components namely; fuzzification, rule base, inference engine and defuzzification. The processes of making crisp inputs are mapped to their fuzzy representation in the process called fuzzification. This involves application of membership functions such as triangular, trapezoidal, Gaussian etc. The inference engine process maps fuzzified inputs to the rule base to produce a fuzzy output. A consequent of the rule and its membership to the output sets are determined here. The defuzzification process converts the output of a fuzzy rule into crisp outputs by one of defuzzification strategies. Thus, fuzzy sets and fuzzy rules together form the knowledge base of a rule-based inference system.

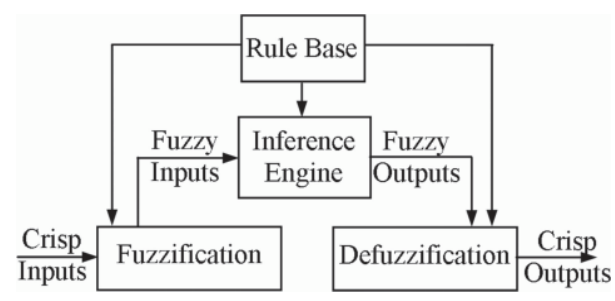

Fig. 5. Typical structure of the fuzzy approach.

Considering a fuzzy system with $\mathrm{p}$ inputs and one output with $\mathrm{M}$ rules, then the $\mathrm{L}_{\text {th }}$ rule has the form [22],[23],[24]: IF $x_{1}$ is $f_{1}^{L}$ and ...xp is $f_{L}^{P} \rightarrow$ Then $y$ is $G^{L}$

\section{A-Star Algorithm}

A-star search algorithm is a widely used graphic searching algorithm. It is also a highly efficient heuristic algorithm used in finding a variable or low cost path. It is considered as one of the best 
intelligent search algorithms that combines the merits of both depth-first search algorithm and breadthfirst algorithm.

A-star path searching algorithm uses the evaluation function (usually denoted $\mathrm{f}(\mathrm{n})$ ) to guide and determine the order in which the search visits nodes in the tree. The evaluation function is given as:

$$
f(n)=g(n)+h(n)
$$

where $g(n)$ is the actual cost from the initial node (start node) to node $n$ (i.e. the cost finding of optimal path), $\mathrm{h}(\mathrm{n})$ is the estimated cost of the optimal path from node $\mathrm{n}$ to the target node (destination node), which depends on the heuristic information of the problem area [25].

Generally, A-star algorithm maintains two lists, an OPEN

List and a CLOSE list. The OPEN list is a priority queue and keeps track of the nodes in it to find out the next node with least evaluation function to pick. The CLOSE list keeps track of nodes that have already been examined. Initially, the OPEN list contains the starting node. When it iterates once, it takes the top of the priority list, and then checks whether it is the goal node (destination node). If so, the algorithm is done. Otherwise, it calculates the evaluation function of all adjacent nodes and adds them to the OPEN list. After the A-star algorithm is completed, it will find a solution if a solution exists. If it doesn't find a solution, then it can guarantee that no such solution exists. A-star algorithm will find a path with the lowest possible cost. This will depend heavily upon the quality of the cost function and estimates provided [26].

A-star algorithm (Pseudo-code A*) may be expressed as following [25], [27]:

Create the open list of nodes, initially containing only our starting node

Create the closed list of nodes, initially empty

While (we have not reached our goal) \{

Consider the best node in the open list (the node with the lowest $f$ value)

If (this node is the goal) \{

Then we're done

\}

Else \{

Move the current node to the closed list and consider all of its neighbors

For (each neighbor) \{

if (this neighbor is in the closed list and our current $g$ value is lower) \{

Update the neighbor with the new, lower, g value

Change the neighbor's parent to our current node

\}

Else if (this neighbor is in the open list and our current $g$ value is lower) \{

Update the neighbor with the new, lower, g value

\}

Change the neighbor's parent to our current node

Else this neighbor is not in either the open or closed list \{

Add the neighbor to the open list and set its g value

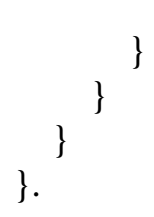

\section{Simple Additive Weighting Methods of Multi Criteria Decision Making}

Various multi-criteria decision making (MCDM) methods have been proposed to solve diverse applications of decision problems. One of the MCDM methods is additive weighting-based method. Simple Additive Weighting (SAW) which is also known as weighted linear combination or scoring methods is a simple and most often used multi attribute decision technique. The method is 
based on the weighted average. An evaluation score is calculated for each alternative by multiplying the scaled value given to the alternative of that attribute with the weights of relative importance directly assigned by decision maker followed by summing of the products for all criteria. The advantage of this method is that it is a proportional linear transformation of the raw data which means that the relative order of magnitude of the standardized scores remains equal. Process of SAW consist of these steps:

1. Create a Decision Matrix according below table and Quantification of Decision Matrix:

Create a decision matrix table of the output fuzzy systems 1,2 and 3 according to the following formula is obtained by replacing the values of the output value aij in the matrix multi-criteria fill in. Options include a list of all neighbors of a sensor node is a matrix of which one is selected in the list by the SAW algorithm and the rest of stay in open list. In our proposed methods uses two methods SAW and Majority Vote to decide three expert systems as follows. In the Majority Vote approach between the votes obtained from expert systems, which one that have the highest value is used to jump as destination node and other nodes are in the open list of A-Star algorithm. In SAW, by calculating the indexes weights, contributed to the decision. SAW due to mathematical models have higher accuracy compared to the Majority Vote.

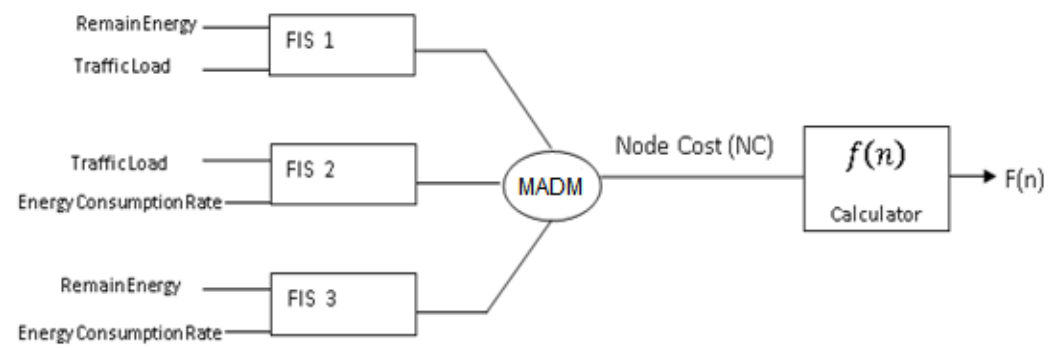

Fig. 6. $A * 3 P F S A W$ and $A * 3 P F M V$ methods of three fuzzy systems with two-parameter mixed by MADM

$$
f(n)=\frac{1}{\operatorname{Min} \operatorname{Hop} \operatorname{Node}(n) \text { to } B S}+N C(n)
$$

n: list of neighboring nodes of a sensor node

Table 1: Multi-Attribute Decision Making Matrix of Scenarios

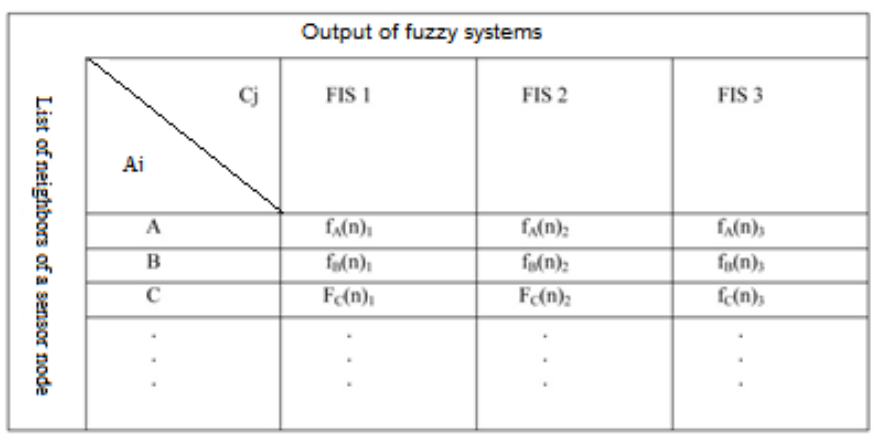

2. Making bi-linear scaling of the values of the Decision Matrix:

For positive indicators: $\quad n_{i j}=\frac{a_{i j}}{\operatorname{Max} a_{i j}} \quad$ For negative indicators: $n_{i j}=1-\frac{a_{i j}}{\operatorname{Max} a_{i j}}$ 
For both positive and negative indicators: $n=\frac{\frac{1}{a_{i j}}}{\operatorname{Max}\left(\frac{1}{a_{i j}}\right)}=\frac{\operatorname{Min} a_{i j}}{a_{i j}}$

3. Multiplying the matrix of weights and measures Scale:

$$
\begin{aligned}
p_{i j} & =\frac{a_{i j}}{\sum_{i=1}^{m} a_{i j}} \quad ; \quad \forall_{j} \\
E_{j} & =-k \sum_{i=1}^{m}\left[p_{i j} \ln p_{i j}\right] \quad ; \quad \forall_{j} \quad k=\frac{1}{\ln (m)} \\
d_{j} & =1-E_{j} ; \quad \forall j \\
w_{j} & =\frac{d_{j}}{\sum_{j=1}^{n} d_{j}} \quad ; \quad \forall_{j}
\end{aligned}
$$

4. Choose the best option (A*) using the following criteria: $A^{*}=\left\{A_{i} \mid \operatorname{Max} \sum_{j=1}^{n} n_{i j} w_{j}\right\}$

\section{PROPOSE D ROUT ING METHOD}

In this paper, the topology of a WSN is modeled as a directed graph $G(N, A)$, where $N$ is the set of nodes, and $A$ is the set of direct links between the nodes. A sink node is responsible for collecting data from all other nodes within its transmission range [5], [9], [10], [26]. The routing schedule is computed by the base station. It calculates optimal routing schedule and broadcasts it. Every node follows this schedule. The process of finding the optimal path, and broadcasting it in the network and sending data from all nodes to the base station by following this routing schedule is repeated in every round. Computation of routing schedule is done dynamically with the consideration of current level of some criteria of each node. For this, normally it may require the nodes to report their criteria periodically to the base station. The base station can then determine the routing schedule based on this updated information.

For the proposed model, whenever any sensor node runs out of energy, communication links between various sensor nodes and the base station will break. This is considered as the end of the network lifetime. Since the lifetime of each sensor node depends on energy consumption, it is important to preserve residual energy of these nodes in such a way that overall network lifetime is extended.

To achieve this goal we propose innovative methods and some of these methods to evaluate the efficiency will be compared. first method is only A-Star algorithm alone which is used as the base routing task, in this way does not consider value of parameters like remaining energy, traffic load and energy consumption tare to select neighbor to jump. In this routing method, the base station prepares the routing schedule and broadcast it to each node. A-star algorithm which is used to find the optimal route from the node to the base station is applied to each node. A-star algorithm creates a tree structure in order to search optimal routing path from a given node to the base station. The tree node is explored based on its evaluation function $f(n)$. The function we used is given as: $f(n)=g(n)+h(n)$.

The second method combines fuzzy methods and the A-star. The tree node is explored based on its evaluation function $f(n)$. The function we used is given as: $f(n)=N C(n)+(1 / M H(n))$. Where $\mathrm{NC}(\mathrm{n})$ is the node cost of node $\mathrm{n}$, which takes value [0...1], and can be calculated by the fuzzy approach. The fuzzy approach is considered for the remaining energy and the traffic load of node $n$ to calculate the optimal cost for node $n$. MH (n) is the short distance from node $n$ to the base station. As a result, the node $n$ that has largest $f(n)$ value will be chosen as the optimal node. 


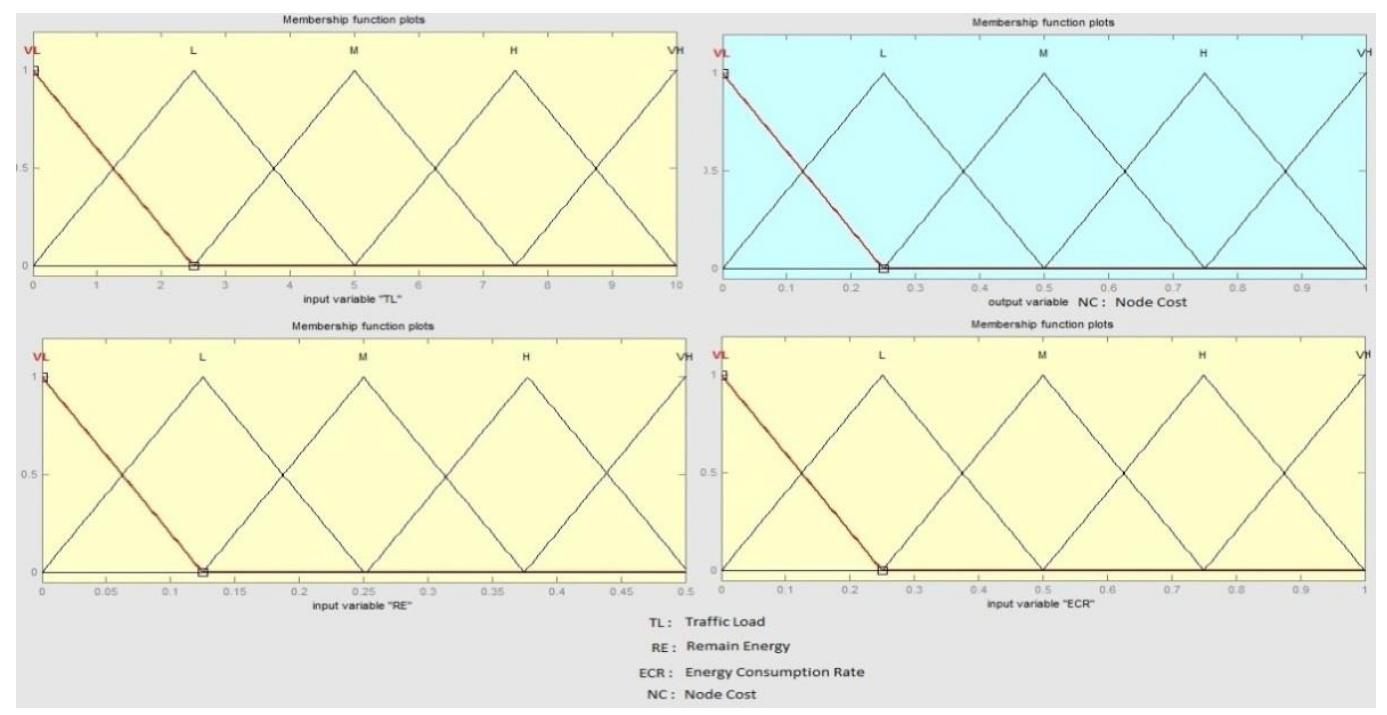

Fig. 7. Membership graph for the inputs (remaining energy, traffic load and energy consumption rate) and the output (node cost).

The goal of the fuzzy part of the proposed protocol is to determine the optimal value of the node cost $\mathrm{NC}(\mathrm{n})$ of node $\mathrm{n}$ that depends on the remaining energy RE(n) and the traffic load TL(n) of node n. Fig. 8 shows the fuzzy approach with two input variables RE(n) and TL(n), and an output NC(n), with universal

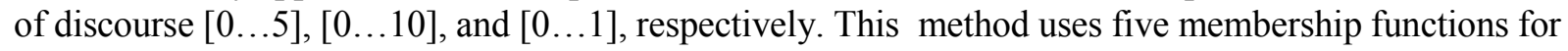
each input and an output variable, as shown in Fig. 7.For the fuzzy approach, the fuzzified values are processed by the inference engine, which consists of a rule base and various methods to inference the rules. The rule base is simply a series of IF-THEN rules that relate the input fuzzy variables and the output variable using linguistic variables each of which is described by fuzzy set and fuzzy implication operator AND. Table A in appendix shows the IF-THEN rules used in the proposed method .All these rules are processed in a parallel manner by a fuzzy inference engine. At the end, the defuzzification finds a single crisp output value from the solution fuzzy space. This value represents the node cost. Practice defuzzification is done using center-of-gravity method [24] given by:

node cost $=\frac{\sum_{i=1}^{n} u_{i} * c_{i}}{\sum_{i=1}^{n} u_{i}}$

Where $\mathrm{Ui}$ is the output of rule base $\mathrm{i}$, and ci is the center of the output membership function.

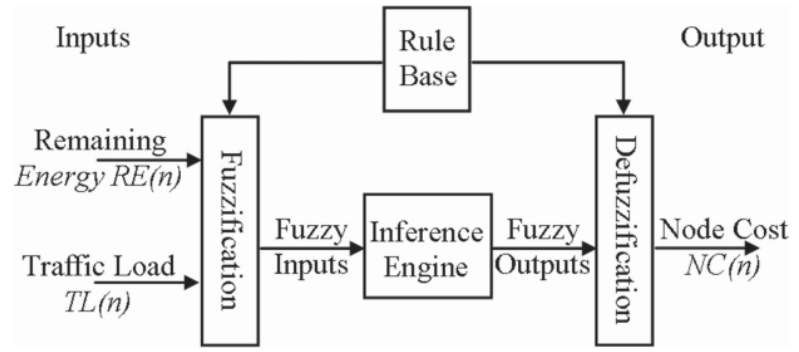

Fig. 8. Fuzzy structure with two inputs (remaining energy and traffic load) and one output (node cost).

A third way to assess the impact of using more parameters in an expert system to decide the choice of the next node in the optimized routing operation uses the third parameter as input of the fuzzy system called the energy consumption rate of the neighbor nodes (ECR). This parameter indicates the use of a 
node in routing process . The more use of nodes in routing process, the less will be used in the selection of preferred nodes as the relay node. Fuzzy rules are attached to the paper. The flowchart of this new method is similar to flowchart of the second method except that the third arguments ECR have also been used in fuzzy systems.

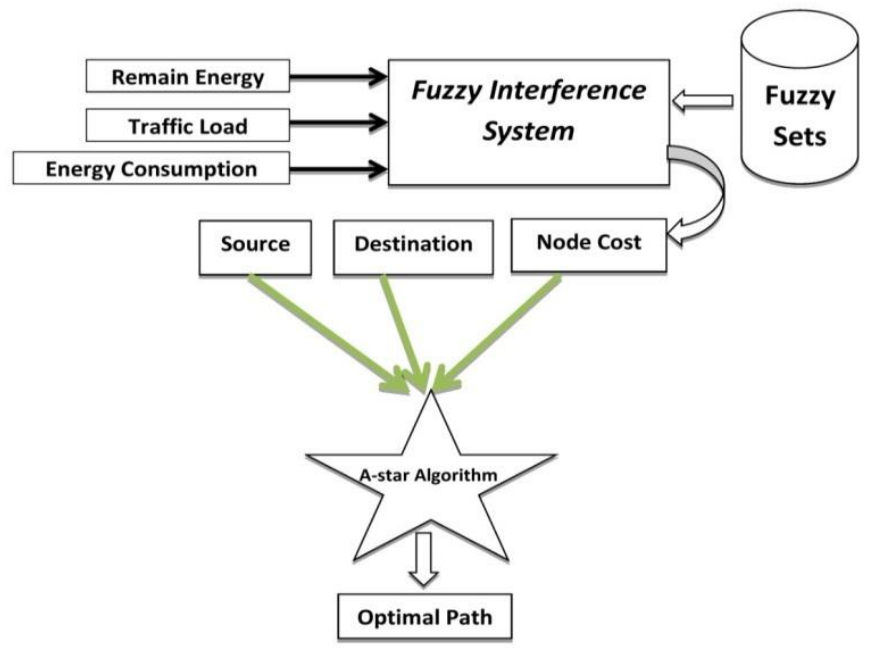

Fig. 9. Two-parameter and three-parameter hybrid algorithm with the A-Star

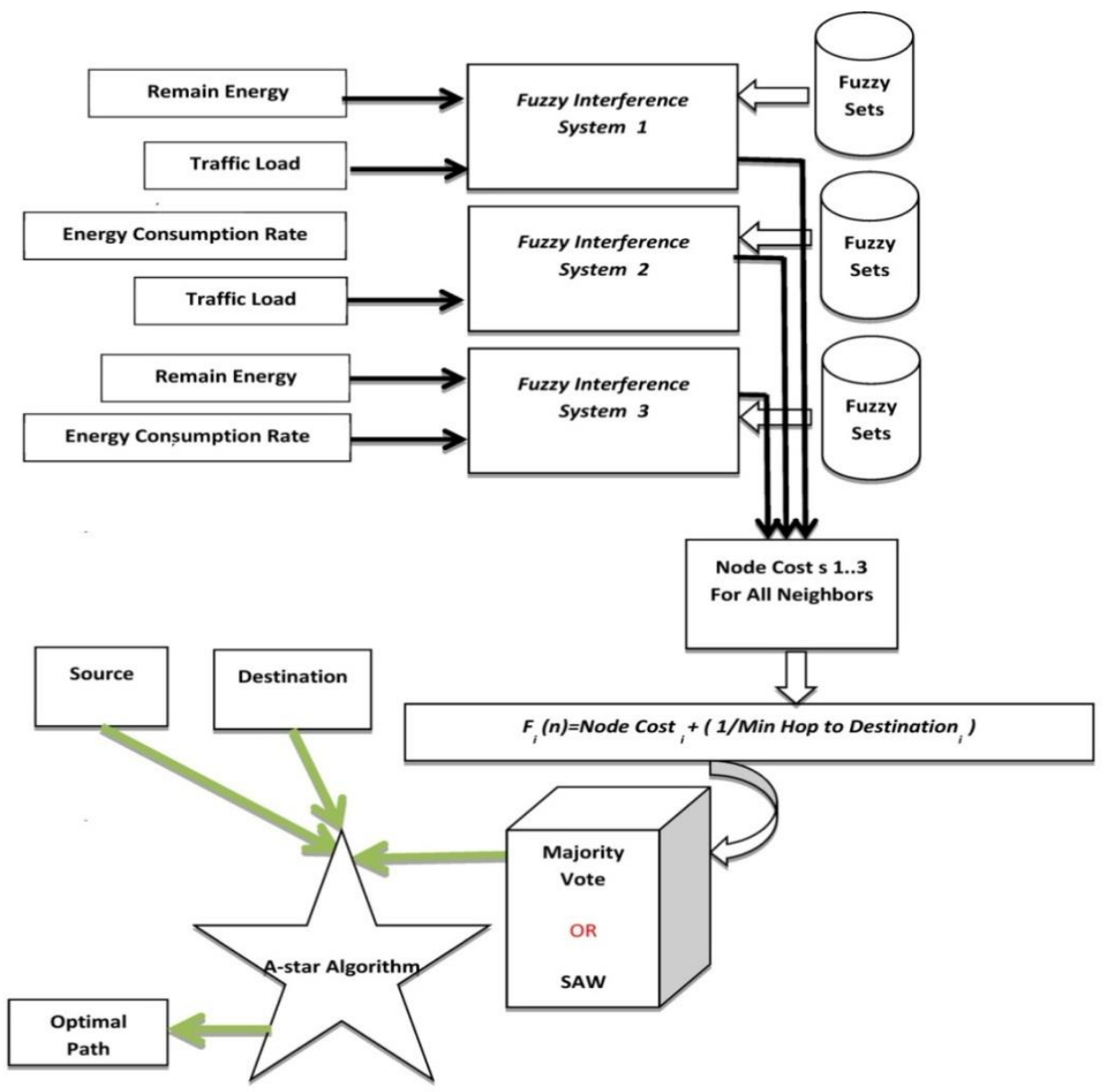

Fig.10. Structure of the other proposed algorithm for routing fuzzy with mixing method of expert systems (SAW or Majority Vote) 
The fourth and fifth proposed methods use several fuzzy expert systems (below chart) by applying Majority vote and SAW methods. In this methods aims to enhance the accuracy of the decision making in choosing the optimal path. In this methods after calculating the minimum number of jumps to the destination $(\mathrm{MH})$ by A-star algorithm, then node cost value calculating by three fuzzy expert systems according to the following chart with the corresponding parameters. Then the values use for calculate $F(n)$ for all neighboring nodes which candidate to jump in order to produce multi-criteria matrix after that SAW algorithm use this matrix to selection optimal neighbor. The Majority Vote approach, instead of a creating multi-criteria matrix to select the best neighbor node for next hop use majority vote Fuzzy expert systems, neighbor node with highest vote will be selected.

\section{PERFORMANCE EVALUATION}

To demonstrate the effectiveness of the proposed methods in terms of balancing energy consumption and maximizing network lifetime, simulation results of the proposed methods are compared with those of A-star search algorithm and with those of Fuzzy mix with A-star (A*2PF) approach and with new methods : Fuzzy 3 parameters mix with A-star $\left(\mathrm{A}^{*} 3 \mathrm{PF}\right)$ and with three-2 parameters Fuzzy systems mix with Astar by Majority Vote (A*3PFMV) and with three-2 parameters Fuzzy systems mix with A-star by SAW (A*3PFSAW), for four different topographical areas according to Table 2.

The simulations are carried out in MATLAB. 100 sensor nodes are randomly deployed in a topographical area A, B, C, D of dimension $100 \mathrm{~m} \times 100 \mathrm{~m}$ and dimension $200 \mathrm{~m} \times 50 \mathrm{~m}$. All topographical areas have the sensed transmission limit of $30 \mathrm{~m}$. The performance of the proposed method is tested in these four topographical areas. There is only one data sink which located at $(90 \mathrm{~m}, 90 \mathrm{~m})$ for area A and at $(180 \mathrm{~m}, 45 \mathrm{~m})$ for area B. All sensor nodes have the same initial energy $0.5 \mathrm{~J}$. The proposed method uses the W.R.Heinzelman radio model that is largely used in the area of routing protocol evaluation in WSNs [28].

$$
\begin{aligned}
& E_{T x}(k, d)=E_{T x-e l e c}(k)+E_{T x-a m p}(k, d)=E_{\text {elec }} * k+\varepsilon_{\text {amp }} * k * d^{2} \\
& E_{R x}(k)=E_{R x-\text { elec }}(k)=E_{\text {elec }} *(k)
\end{aligned}
$$

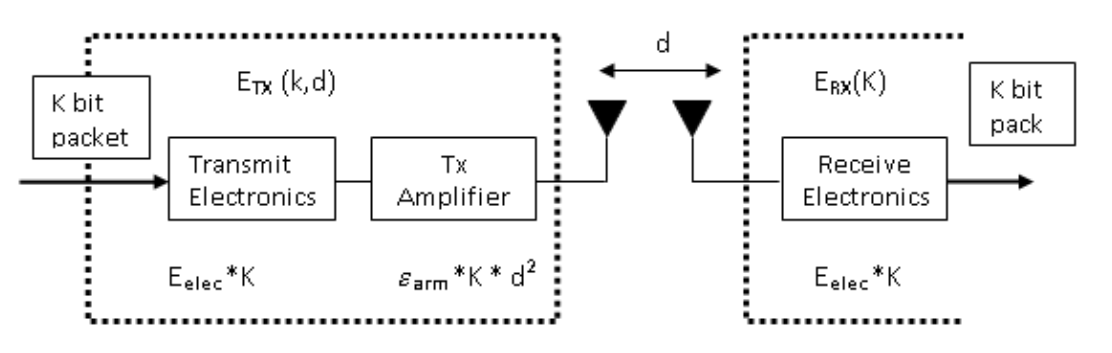

Fig .11. Diagram of the transmitter and receiver wireless sensor

According to this model, transmission and receiving costs are characterized by the expressions $E_{T x}(k, d)$ and $E_{R x}(k)$, respectively, where $\mathrm{k}$ is the number of bit per packet, d is the distance from the sender node to the receiver node, $E_{\text {elec }}$ and $E_{\text {amp }}$ are per bit energy dissipation in transmitting or receiving circuitry and energy required per bit per meter square for the amplifier to achieve acceptable signal to noise ratio (SNR) respectively. Simulations are done using the values $50 \mathrm{~nJ} / \mathrm{bit}$ and $100 \mathrm{pJ} / \mathrm{bit} / \mathrm{m} 2$ for $\mathrm{E}_{\text {elec }}$ and $\mathrm{E}_{\mathrm{amp}}$, respectively. The traffic load, in each node is assumed to be generated randomly between [0...10]. Table 2 presents the systems parameters in details.

Table. 2. SIMULATION PARAMETERS 


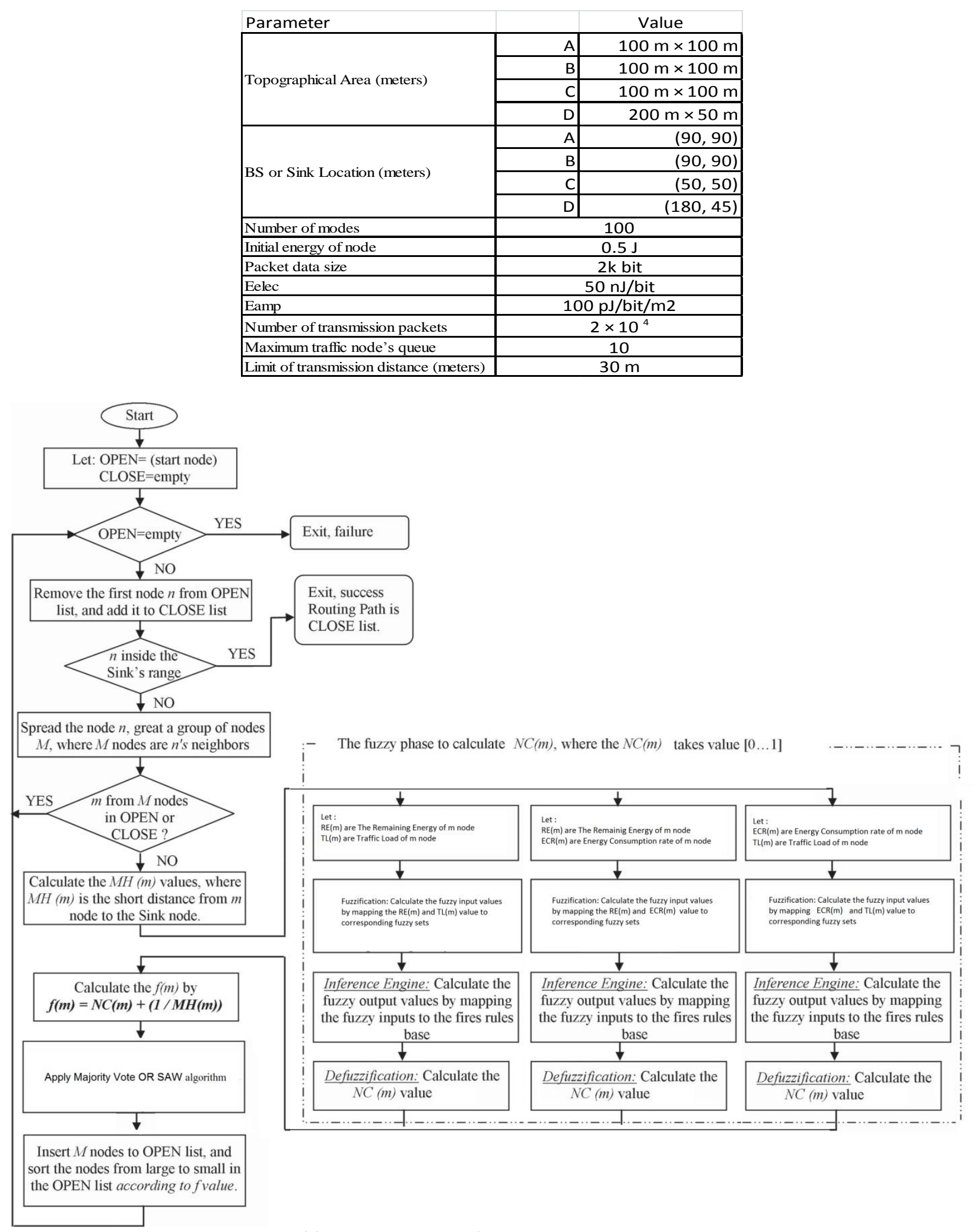

Fig. 12. Flow chart of the proposed algorithms

The proposed models assume that the network has the following features: 
- Sensor nodes and base station networks are static and immobile.

- Links between nodes are symmetric and approximate distance between each node can be calculated based on the received signal strength (RSSI).

- Flat tracking and routing regardless of make and hierarchical cluster heads is performed.

- All nodes are homogeneous and are identical.

- After calculating the location of every sensor nodes, they are stored within each node.

- All sensor nodes are randomly distributed in the environment.

- All sensor nodes have an equal maximum transmission range and initial energy.

- Each sensor node is waiting to receive a certain amount of traffic in its queue. This queue includes Common applications, traffic that receives to send.

- Channel access methods and data management in wireless sensor network nodes is based on the TDMA model, so that each sensor node send data in its time slot that allocated.

\section{METHODS COMPARISION}

As Gaikwad method presented in [29] ,the combination of A-Star and two-parameter Fuzzy results in better performance and increase the lifetime of the network ,for this reason we compare it with our proposed techniques ,and use factors such as: number of living nodes, the total energy consumed in each round of data transmission networks, energy consumption at each network data transmission, the residual energy of node and the total amount of packets in the network in order to evaluate and compare at moment of death.

Table .3. The results of the simulation

\begin{tabular}{|c|c|c|c|c|c|c|c|c|c|c|c|c|c|}
\hline & & \multicolumn{3}{|c|}{ (1)Area $=100 \times 100, B S=90,90$} & \multicolumn{3}{|c|}{ (2)Area $=100 \times 100, B S=90,90$} & \multicolumn{3}{|c|}{ Area $=100 \times 100, B S=50,50$} & \multicolumn{3}{|c|}{ Area $=200 \times 50, B S=180,45$} \\
\hline No & & $\begin{array}{l}\text { First } \\
\text { Dead } \\
\text { Node } \\
\end{array}$ & $\begin{array}{l}\text { Alive } \\
\text { Node }\end{array}$ & \begin{tabular}{|l|} 
Sum \\
Of \\
Packet
\end{tabular} & \begin{tabular}{|l} 
First \\
Dead \\
Node \\
\end{tabular} & $\begin{array}{l}\text { Alive } \\
\text { Node }\end{array}$ & \begin{tabular}{|l} 
Sum \\
Of \\
Packet \\
\end{tabular} & \begin{tabular}{|l} 
First \\
Dead \\
Node \\
\end{tabular} & Alive Node & $\begin{array}{l}\text { Sum } \\
\text { Of } \\
\text { Packet }\end{array}$ & \begin{tabular}{|l} 
First \\
Dead \\
Node \\
\end{tabular} & $\begin{array}{l}\text { Alive } \\
\text { Node }\end{array}$ & \begin{tabular}{|l|} 
Sum \\
Of \\
Packet \\
\end{tabular} \\
\hline 1 & A-Star & 12952 & 70 & 15875 & 16082 & 71 & 20907 & 25555 & 77 & 32696 & 6967 & 72 & 9775 \\
\hline 2 & $\begin{array}{l}\text { A-Star 2P } \\
\text { Fuzzy }\end{array}$ & 17664 & 68 & 18679 & 22952 & 61 & 23605 & 31584 & 72 & 34477 & 10753 & 76 & 11608 \\
\hline 3 & $\begin{array}{l}\text { A-Star 3P } \\
\text { Fuzzy }\end{array}$ & 16695 & 82 & 18650 & 17672 & 90 & 22571 & 27513 & 84 & 34404 & 8461 & 96 & 9987 \\
\hline 4 & $\begin{array}{l}\text { A-Star 3PF } \\
\text { Majority } \\
\text { Vote }\end{array}$ & 17537 & 65 & 18714 & 21638 & 49 & 23943 & 32385 & 51 & 34495 & 9523 & 80 & 11868 \\
\hline 5 & $\begin{array}{l}\text { A-Star 3PF } \\
\text { SAW }\end{array}$ & 17424 & 57 & 18730 & 22191 & 36 & 23989 & 31269 & 44 & 34626 & 10828 & 81 & 11914 \\
\hline
\end{tabular}

What the chart comparison charts and the rate of improvement and the results of simulations in four topographical environment achieved indicate that in spite of the very small decreasing network lifetime compare to the proposed methods [29], we see a remarkable improvement in the number of nodes alive in A-Star combine by fuzzy with three parameters due to the impact of the rate of energy consumption in routing. A few declines in the network lifetime is due to the efforts of the algorithm in reducing the rate of energy consumption in the nodes with the possibility of choosing a long routes. But at a time, we want to add new BS network in the scenario, due to more nodes alive, A*3PF would be the best way.

Second and third new methods have been presented in this paper that the decision for choosing the best path with regard to energy consumption rate using mix of expert systems with majority of the votes and simple adaptive weight will improve performance and increase network lifetime and this is because using more effective energy consumption rate parameter. Although number of alive nodes a little bit less but this is not the remarkable decline. Between the last two methods of mixing expert systems method, simple adaptive weight (SAW), due to having precise mathematical model, we see clearer and better 
improvement. Overall on condition that the aim is the more survival of the network and more data transmission below methods are optimal are the following respectively: $A * 3 P F S A W, A * 3 P F M V, A * 2 P F$, $\mathrm{A} * 3 \mathrm{PF}, \mathrm{A}^{*}$. And in case you want to have more vivid node, the best way to phase three parameters $\left(\mathrm{A}^{*} 3 \mathrm{PF}\right)$
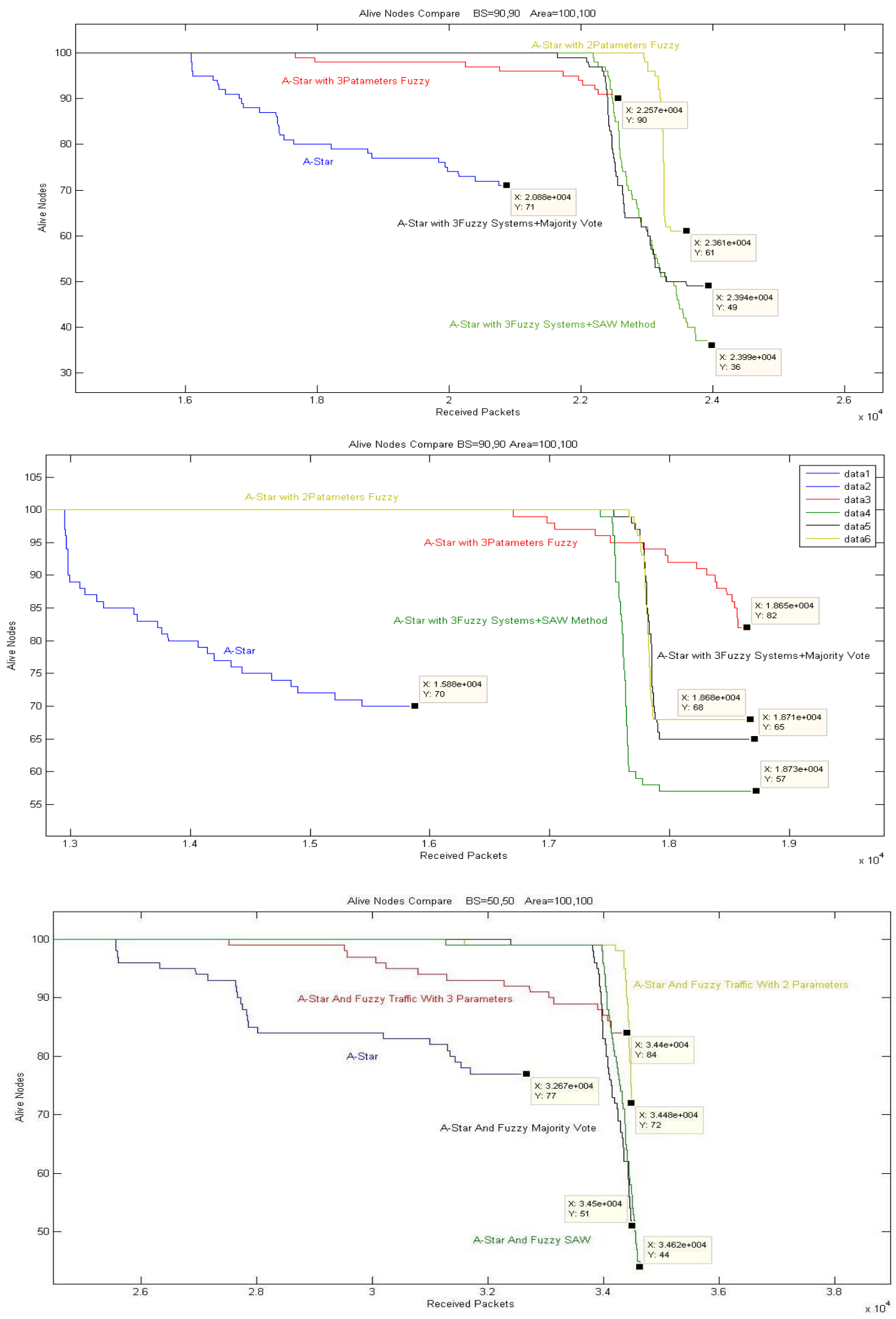


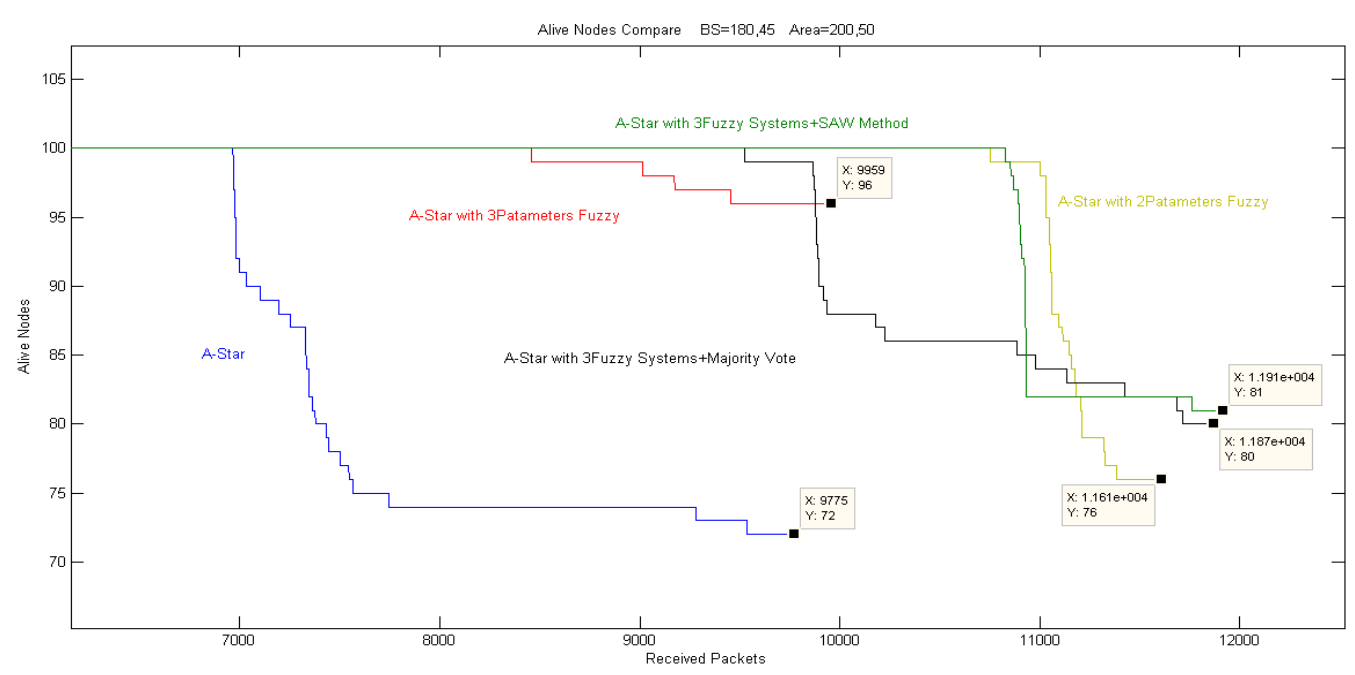

Fig. 13. Comparison chart of the five algorithms in four topographic areas

Table .4. Improvement of the proposed methods in comparison with the $\mathrm{A}^{*} 2 \mathrm{PF}$

\begin{tabular}{|c|c|c|c|c|c|c|c|c|c|c|c|c|}
\hline & \multicolumn{6}{|c|}{ (1)Area $=100 \times 100, B S=90,90$} & \multicolumn{6}{|c|}{ (2)Area $=100 \times 100, B S=90,90$} \\
\hline & $\begin{array}{l}\text { First } \\
\text { Dead } \\
\text { Node }\end{array}$ & Improvement & $\begin{array}{l}\text { Alive } \\
\text { Node }\end{array}$ & Improvement & $\begin{array}{l}\text { Sum } \\
\text { of } \\
\text { Packet }\end{array}$ & Improvement & $\begin{array}{l}\text { First } \\
\text { Dead } \\
\text { Node }\end{array}$ & Improvement & $\begin{array}{l}\text { Alive } \\
\text { Node }\end{array}$ & Improvement & $\begin{array}{l}\text { Sum } \\
\text { Of } \\
\text { Packet }\end{array}$ & Improvement \\
\hline $\begin{array}{l}\text { A-Star 2P } \\
\text { Fuzzy }\end{array}$ & 17664 & - & 68 & - & 18679 & - & 22952 & - & 61 & - & 23605 & - \\
\hline $\begin{array}{l}\text { A-Star 3P } \\
\text { Fuzzy }\end{array}$ & 16695 & $5.49-$ & 82 & 20.59 & 18650 & $0.16-$ & 17672 & 23- & 90 & 47.54 & 22571 & 4.38- \\
\hline $\begin{array}{l}\text { A-Star 3PF } \\
\text { Majority } \\
\text { Vote }\end{array}$ & 17537 & $0.72-$ & 65 & $4.41-$ & 18714 & 0.19 & 21638 & $5.72-$ & 49 & 19.67- & 23943 & 1.43 \\
\hline $\begin{array}{l}\text { A-Star 3PF } \\
\text { SAW }\end{array}$ & 17424 & $1.36-$ & 57 & 16.17- & 18730 & 0.27 & 22191 & $3.32-$ & 36 & $40.98-$ & 23989 & 1.63 \\
\hline
\end{tabular}

\begin{tabular}{|c|c|c|c|c|c|c|c|c|c|c|c|c|}
\hline & \multicolumn{6}{|c|}{ Area $=100 \times 100, B S=50,50$} & \multicolumn{6}{|c|}{ Area $=200 \times 50, B S=180,45$} \\
\hline & $\begin{array}{l}\text { First } \\
\text { Dead } \\
\text { Node }\end{array}$ & Improvement & $\begin{array}{l}\text { Alive } \\
\text { Node }\end{array}$ & Improvement & $\begin{array}{l}\text { Sum } \\
\text { Of } \\
\text { Packet }\end{array}$ & Improvement & $\begin{array}{l}\text { First } \\
\text { Dead } \\
\text { Node } \\
\end{array}$ & Improvement & $\begin{array}{l}\text { Alive } \\
\text { Node }\end{array}$ & Improvement & $\begin{array}{l}\text { Sum } \\
\text { Of } \\
\text { Packet } \\
\end{array}$ & Improvement \\
\hline $\begin{array}{l}\text { A-Star 2P } \\
\text { Fuzzy }\end{array}$ & 31584 & - & 72 & - & 34477 & - & 10753 & - & 76 & - & 11608 & - \\
\hline $\begin{array}{l}\text { A-Star 3P } \\
\text { Fuzzy }\end{array}$ & 27513 & $12.89-$ & 84 & 16.67 & 34404 & $0.21-$ & 8461 & 21.31- & 96 & 26.32 & 9987 & $13.96-$ \\
\hline $\begin{array}{l}\text { A-Star 3PF } \\
\text { Majority } \\
\text { Vote }\end{array}$ & 32385 & 2.54 & 51 & 29.17- & 34495 & 0.05 & 9523 & 11.44- & 80 & 5.26 & 11868 & 2.24 \\
\hline $\begin{array}{l}\text { A-Star 3PF } \\
\text { SAW }\end{array}$ & 31269 & $1-$ & 44 & 38.89- & 34626 & 0.43 & 10828 & 0.7 & 81 & 6.58 & 11914 & 2.64 \\
\hline
\end{tabular}




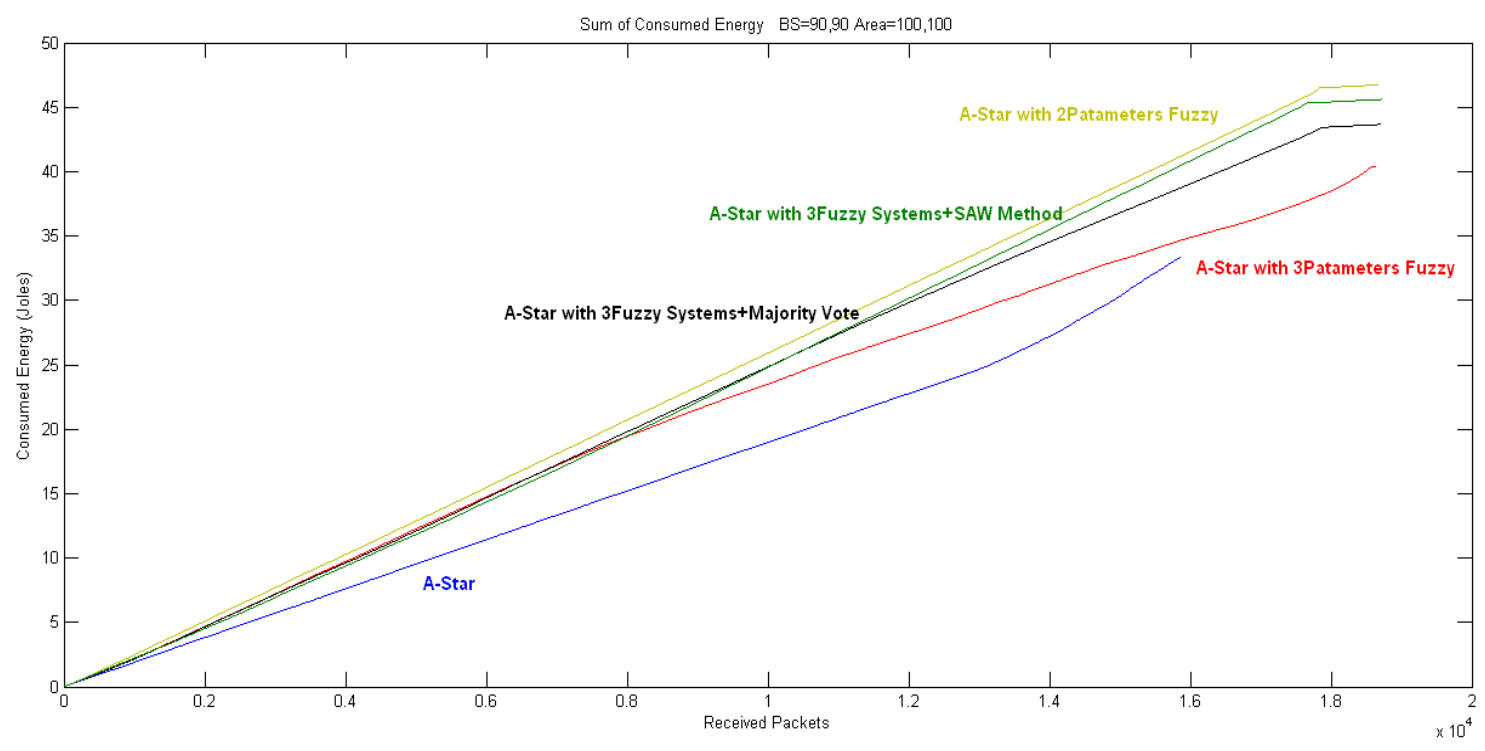

Fig .14. Total network energy consumption in each round of data transmission

Chart above, the total energy consumed in the network is sending data. The lower slope indicates a lower power consumption rate for sending data. So A-Star method initially acts very well, and for sending data energy has low consumption rate, but in the end, it has high slope in energy consumption and will be more than the other methods, which causes more and rapid consumption of energy and therefore nodes in the network will die sooner. Fuzzy method with two parameters has the highest rate of energy consumption. Since the rate of energy consumption is not involved in the selection of the route, so it's pretty uniform and totally linear in power consumption curve. But in the Majority Vote and SAW method with balanced energy consumption, we witness increasing in network lifetime and more data sent. In $\mathrm{A} * 3 \mathrm{PF}$ approach the more pass network lifetime, the more changing in energy consumption rate and this approach adapt itself to the conditions, so the number of alive nodes in the end will be more.

The below graphs represent the amount of energy nodes in the network death time. It shows that network in the $\mathrm{A}^{*}$ method will die while many nodes are still high energy and alive In $\mathrm{A}$ *2PF approach many nodes die while than next method less packets sent, but in A*3PF, A*3PFSAWand A*3PFMV approaches the situation is better, the nodes with high energy vs. the nodes with low energy is almost balanced. The balances of energy consumption of all nodes in the network have been done better. The Majority Vote and SAW methods regardless of the increasing network lifetime, we will see balance in the energy consumption at each round, which indicates that these two methods is better.

To sum up of comparisons it can be said that the death of the first node and the number of nodes remaining alive in the last round and the remaining amount of energy networks in the different algorithms are affected by some factors like: the movement of the geographical location of the BS and change the size of the network environment and make different network behavior. 

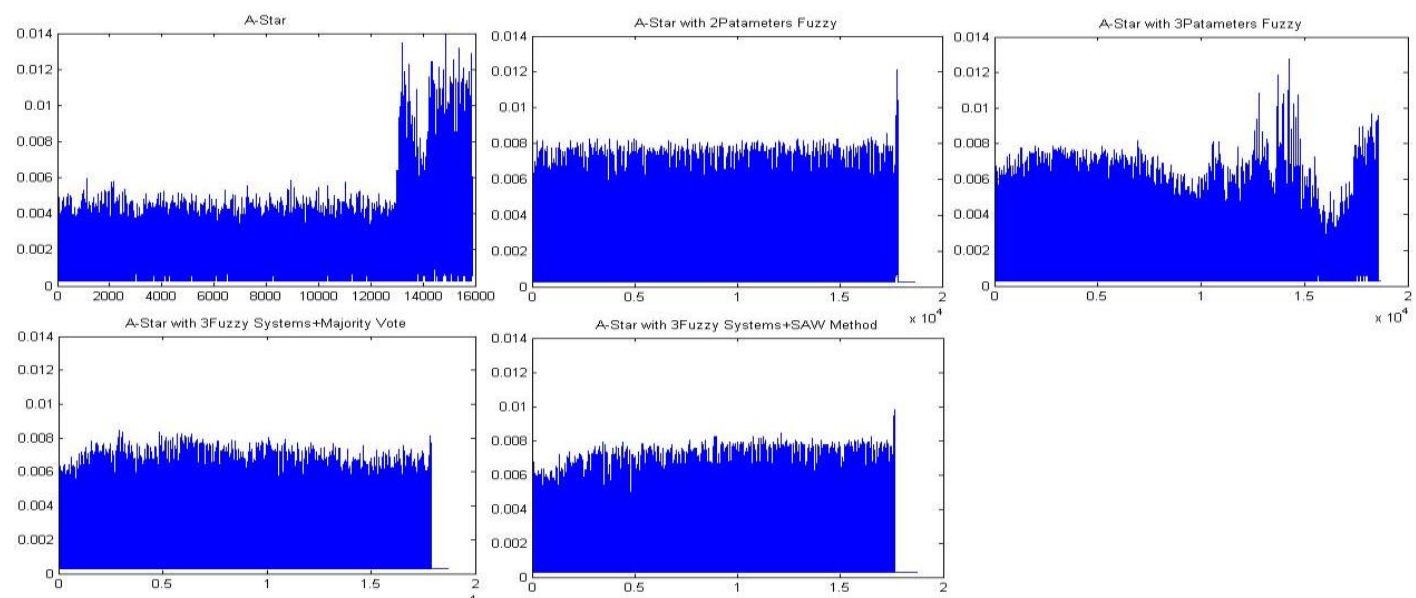

Fig. 15. Amount of network energy used in each time of data transmission (round)
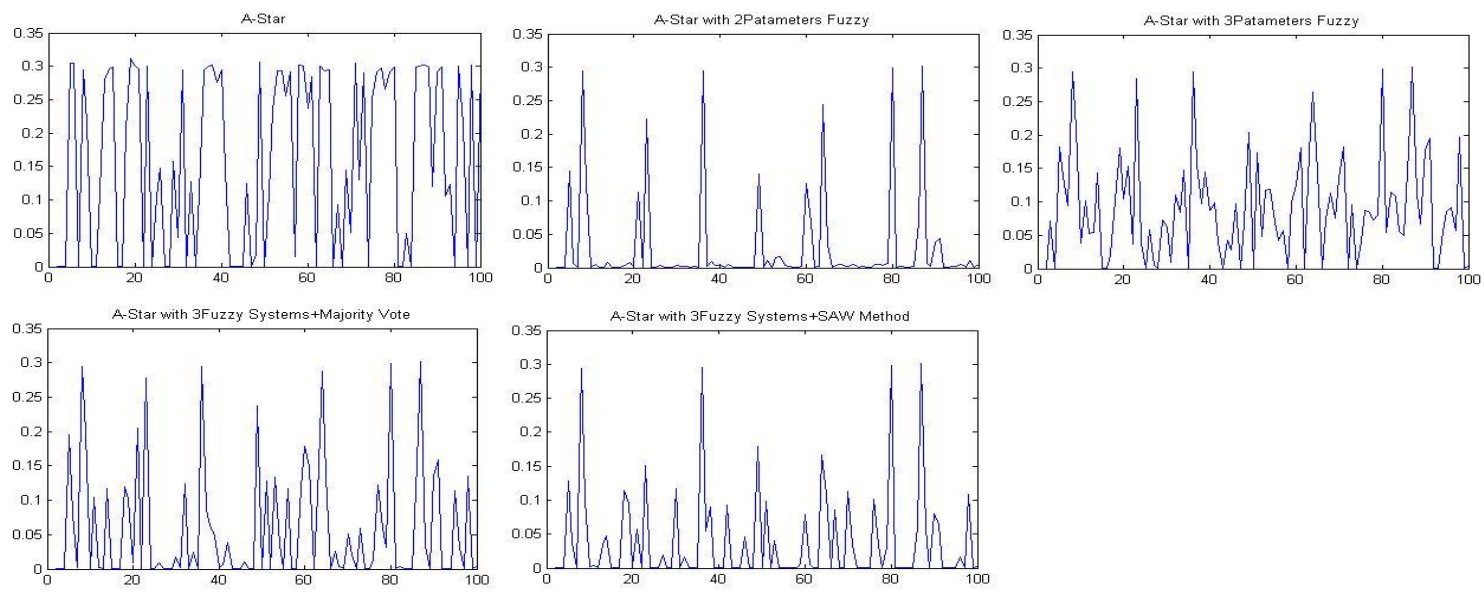

Fig. 16. Energy levels of network nodes at the moment of death.

\section{CONCLUSION}

In wireless sensor networks where nodes operate on limited battery energy efficient utilization of the energy is very important. One of the main characteristics of these networks is that the network lifetime is highly related to the route selection. Unbalanced energy consumption is an inherent problem in a WSN. To efficiently route data through transmission path from node to node and to prolong the overall lifetime of the network, we proposed some new algorithms by using a combination of both Mix-Fuzzy approach and A-star algorithm. The new method is capable of selecting optimal routing path from the source node to the sink by favoring the highest remaining energy, minimum number of hops, lowest traffic load and lowest energy consumption rate. The performance of the proposed method is evaluated and compared with other methods under the same criteria in four different topographical areas. By using mix of expert system and proper use of three parameters: remaining energy, traffic load and energy consumption rate in their fuzzy system Simulation results demonstrate the effectiveness of the new approaches -A*3PFSAW and $\mathrm{A} * 3 \mathrm{PFMV}$ - than $\mathrm{A} * 2 \mathrm{PF}, \mathrm{A} * 3 \mathrm{PF}, \mathrm{A} *$ methods with regards to enhancement of the lifetime of wireless sensor networks with randomly scattered nodes.

However according the results and collected statistics should be noted that the network performance improvement in terms of the maximizing lifetime will be affected by such as geographical placement BS 
and the size (length and width) of the network, the number of sensor nodes, neighborhood radius, BS and node mobility, the amount of initial network energy, heuristic algorithm, the type of algorithm used for mixing experts systems ,nodes distribution in the network environment, node density, etc. but to sum up, our new proposed algorithms - A*3PFSAW, A*3PFMV - in the improvement of performance and network lifetime for different scenarios implemented have better performance and stability of the algorithm in such a network.

Table .5. Summary of the proposed methods in comparison

\begin{tabular}{|c|c|c|c|c|}
\hline & \multicolumn{4}{|c|}{ First Dead Node } \\
\hline Rank $T$ & Topography Area 100100 - BS 9090 & Topography Area 100100 - BS 90 90-New Initial & Topography Area 100100 - BS 5050 & Topography Area 200 50 - BS 18045 \\
\hline 1 & \multicolumn{2}{|c|}{ A-Star with 2Patameters Fuzzy } & A-Star with 3Fuzzy Systems+Majority Vote & A-Star with 3Fuzzy SystemstSAW Method \\
\hline & 2 A-Star with 3Fuzzy Systems+Majority Vote & A-Star with 3Fuzzy Systems+SAW Method & \multicolumn{2}{|c|}{ A-Star with 2Patameters Fuzzy } \\
\hline & A-Star with 3Fuzzy SystemstSAW Method & A-Star with 3Fuzzy Systems+Majority Vote & A-Star with 3Fuzzy Systems+SAW Method & A-Star with 3Fuzzy Systems+Majority Vote \\
\hline 4 & \multicolumn{4}{|c|}{ A-Star with 3Patameters Fuzzy } \\
\hline 5 & \multicolumn{4}{|c|}{ A-Star } \\
\hline & \multicolumn{4}{|c|}{ Alive Nodes on Last state } \\
\hline Rank $T$ & Topography Area 100100 - BS 9090 & Topography Area 100100 - BS 90 90-New Initial & Topography Area 100100 - BS 5050 & Topography Area 200 50 - BS 18045 \\
\hline 1 & \multicolumn{4}{|c|}{ A-Star with 3Patameters Fuzzy } \\
\hline 2 & \multicolumn{3}{|c|}{ A-Star } & A-Star with 3Fuzzy Systems+SAW Method \\
\hline 3 & \multicolumn{3}{|c|}{ A-Star with 2Patameters Fuzzy } & A-Star with 3Fuzzy Systems+Majority Vote \\
\hline 4 & \multicolumn{3}{|c|}{ A-Star with 3Fuzzy Systems+Majority Vote } & A-Star with 2Patameters Fuzzy \\
\hline 5 & \multicolumn{3}{|c|}{ A-Star with 3Fuzzy Systems+SAW Method } & A-Star \\
\hline & \multirow{2}{*}{\multicolumn{4}{|c|}{ Sum of Packet Sent (Life Time Of The Network) }} \\
\hline & & & & \\
\hline Rank 1 & Topography Area 100100 - BS 9090 & Topography Area 100100 - BS 90 90-New Initial & Topography Area 100100 - BS 5050 & Topography Area 200 50 - BS 18045 \\
\hline 1 & \multicolumn{4}{|c|}{ A-Star with 3Fuzzy Systems+SAW Method } \\
\hline 2 & \multicolumn{4}{|c|}{ A-Star with 3Fuzzy Systems+Majority Vote } \\
\hline 3 & \multicolumn{4}{|c|}{ A-Star with 2Patameters Fuzzy } \\
\hline 4 & \multicolumn{4}{|c|}{ A-Star with 3Patameters Fuzzy } \\
\hline 5 & \multicolumn{4}{|c|}{ A-Star } \\
\hline
\end{tabular}

\section{References}

1- R. V. Kulkarni, A. Forster, and G. K. Venayagamoorth, "Computational intelligence in wireless sensor networks: A survey," IEEE Commun. Surveys Tutorials, vol. 13, no. 1, pp. 68-96, Feb. 2011.

2- C. Hua and T. P. Yum, "Optimal routing and data aggregation for maximizing lifetime of wireless sensor networks," IEEE ACM Trans. Netw., vol. 16, no. 4, pp. 892-903, Aug. 2008.

3- H. Zhang and H. Shen, "Balancing energy consumption to maximize network lifetime in data-gathering sensor networks," IEEE Trans. Par- allel Distrib. Syst., vol. 20, no. 10, pp. 1526-1539, Oct. 2009.

4- J. N. Al-Karaki and A. E. Kamal, "Routing techniques in wireless sensor networks: A survey," IEEE Wireless Commun., vol. 11, no. 6, pp. 6-28, Dec. 2004.

5- H. R. Karkvandi, E. Pecht, and O. Yadid, "Effective lifetime-aware routing in wireless sensor networks," IEEE Sensors J., vol. 11, no. 12, pp. 3359-3367, Dec. 2011.

6- K. Akkaya and M. Younis, "A survey of routing protocols in wireless sensor networks," Ad Hoc Netw., vol. 3, no. 3, pp. 325-349, May 2005.

7- F. Ren, J. Zhang, T. He, C. Lin, and S. K. Das, "EBRP: Energy-balanced routing protocol for data gathering in wireless sensor networks," IEEE Trans. Parallel Distrib. Syst., vol. 22, no. 12, pp. 2108-2125, Dec. 2011.

8- M. J. Tsai, H. Y. Yang, and W. Q. Huang, "Axis-based virtual coordinate assignment protocol and deliveryguaranteed routing protocol in wireless sensor networks," in Proc. IEEE INFOCOM 26th Int. Conf. Comput. Commun. May 2007, pp. 2234-2242. 
9- J. Park and S. Sahni, "An online heuristic for maximum lifetime routing in wireless sensor networks," IEEE Trans. Comput., vol. 55, no. 8, pp.1048-1056, Aug. 2006.

10- C. Wu, R. Yuan, and H. Zhou, "A novel load balanced and lifetime maximization routing protocol in wireless sensor networks," in Proc. IEEE Vehicular Technol. Conf. VTC Spring, May 2008, pp. 113-117.

11- C. C. Hung, K. C. J. Lin, C.-C. Hsu, C.-F. Chou, and C.-J. Tu, "On enhancing network-lifetime using opportunistic routing in wireless sensor networks," in Proc. 19th Int. Conf. Comput. Commun. Netw, Aug. 2010, pp. 1-6.

12- R. Madan and S. Lall, "Distributed algorithms for maximum lifetime routing in wireless sensor networks," IEEE Trans. Wireless Commun., vol. 5, no. 8, pp. 2185-2193, Aug. 2006.

13- J. H. Chang and L. Tassiulas, "Maximum lifetime routing in wireless sensor networks," IEEE/ACM Trans. Netw., vol. 12, no. 4, pp. 609-619, Aug. 2004.

14- O. Zytoune, M. El-Aroussi, and D. Aboutajdine, "A uniform balancing energy routing protocol for wireless sensor networks," Wireless Personal Commun. vol. 55, no. 2, pp. 147-161, Oct. 2010.

15- Y. M. Lu and V. W. S. Wong, "An energy-efficient multipath routing protocol for wireless sensor networks," in Proc. IEEE 64th Vehicular Technol. Conf., Sep. 2006, pp. 1-5.

16- C. Park and I. Jung, "Traffic-aware routing protocol for wireless sensor networks," in Proc. IEEE Inform. Sci. Appl. Int. Conf., Apr. 2010, pp.1-8.

17- M. R. Minhas, S. Gopalakrishnan, and V. C. M. Leung, "An online multipath routing algorithm for maximizing lifetime in wireless sensor networks," in Proc. IEEE Inform. Technol. New Generat. 6th Int. Conf., Apr. 2009, pp. 581-586.

18- M. A. Azim and A. Jamalipour, "Performance evaluation of optimizedforwarding strategy for flat sensor networks," in Proc. IEEE GlobalTelecommun. Conf., Nov. 2007, pp. 710-714.

19- S. Y. Chiang and J. L. Wang, "Routing analysis using fuzzy logicsystems in wireless sensor networks," Lecture Notes Comput. Sci., vol.5178, pp. 966-973, 2008.

20- K. M. Rana and M. A. Zaveri, "ASEER: A routing method to extend life of two-tiered wireless sensor network," Int. J. Adv. Smart Sensor Netw. Syst., vol. 11, no. 2, pp. 1-16, Oct. 2011.

21- W. Dargie and C. Poellabauer, "Network layer," in Fundamental of Wireless Sensor Networks Theory and Practice. New York: Wiley, 2010, pp. 163-204.

22- L. A. Zadeh, "Soft computing and fuzzy logic," IEEE Software, vol. 11, no. 6, pp. 48-56, Nov. 1994.

23- K.-Y. Cai and L. Zhang, "Fuzzy reasoning as a control problem," IEEETrans. Fuzzy Syst., vol. 16, no. 3, pp. 600614, Jun. 2008.

24- T. A. Runkler, "Selection of appropriate defuzzification methods using application specific properties," IEEE Trans. Fuzzy Syst., vol. 5, no. 1, pp. 72-79, Feb. 1997.

25- K. M. Passino and P. J. Antsaklis, "A metric space approach to the specification of the heuristic function for the A* algorithm," IEEE Trans. Syst. Man Cybern., vol. 24, no. 1, pp. 159-166, Jan. 1994.

26- X. H. Li, S. H. Hong, and K. L. Fang, "WSNHA-GAHR: A greedy and A* heuristic routing algorithm for wireless sensor networks in home automation," IET Commun., vol. 5, no. 13, pp. 1797-1805, Sep. 2011.

27- J. Yao, C. Lin, X. Xie, A. J. Wang, and C. C. Hung, "Path planning forvirtual human motion using improved A* star algorithm," in Proc. IEEE Inform. Technol. New Generat. 7th Int. Conf., Apr. 2010, pp. 1154-1158.

28- W. Heinzelman, A. Chandrakasan and H. Balakrishnan, "Energy-Efficient Communication Protocol for Wireless Mi- crosensor Networks," Proceedings of the 33rd Hawaii International Conference on System Sciences (HICSS '00), January 2000.

29- Deepak S. Gaikwad, Sampada Pimpale, Routing Alternatives for Network Lifetime Maximization of WSNs Using Heuristic and Fuzzy Logic Approach , International Journal of Inventive Engineering and Sciences (IJIES) ISSN: 2319-9598, Volume-1, Issue-6, May 2013 


\section{APPENDIX}

Table A: Fuzzy Rules
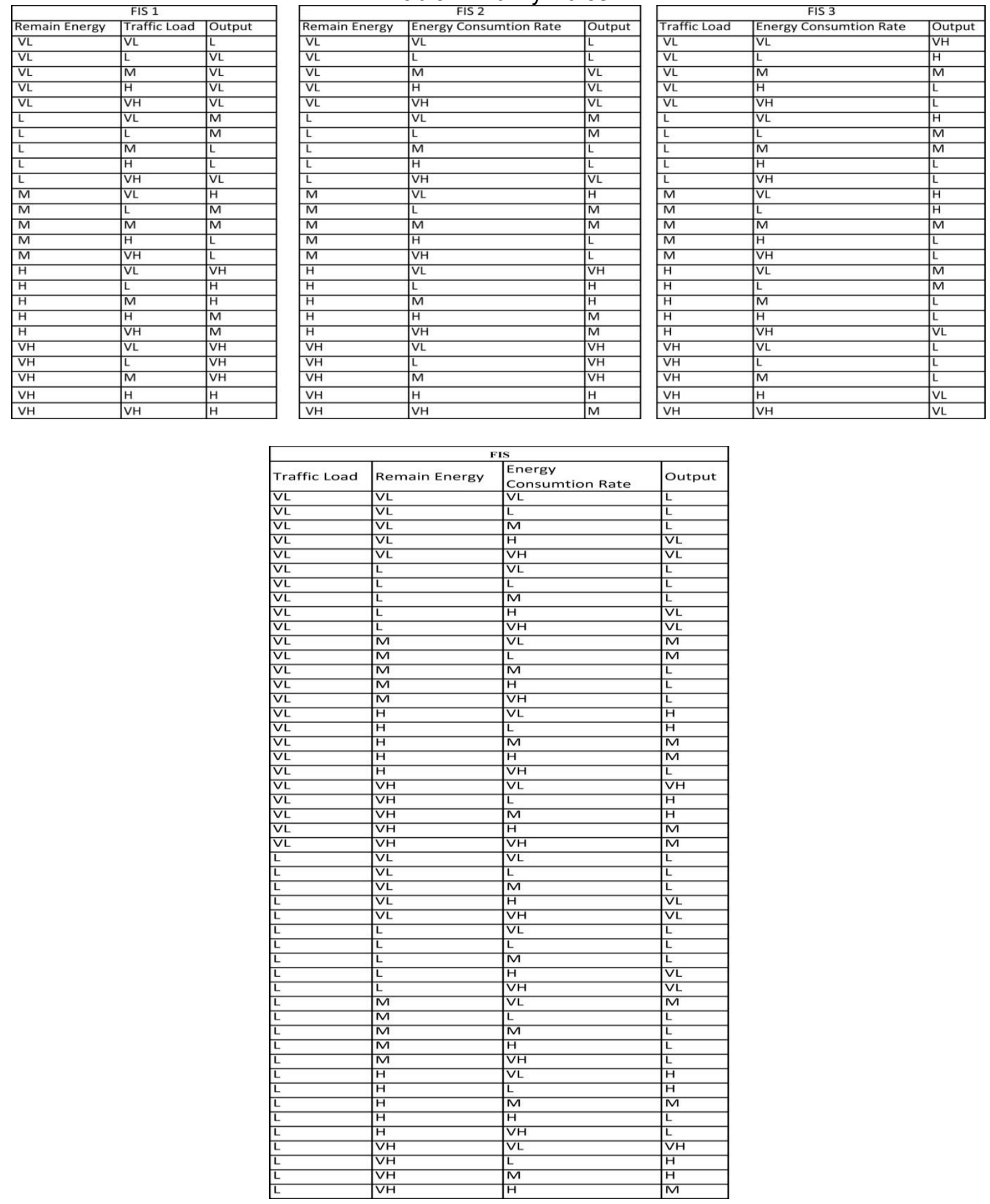